\title{
The most negative ion in the Thomas-Fermi-von Weizsäcker theory of atoms and molecules
}

\author{
Rafael Benguria $\uparrow \S \|$ and Elliott $\mathrm{H}$ Lieb $\ddagger \|$ \\ † Departamento de Física, Universidad de Chile, Casilla 5487, Santiago, Chile \\ $\ddagger$ Departments of Mathematics and Physics, Princeton University, PO Box 708, Princeton, \\ NJ 08544, USA
}

Received 4 May 1984

\begin{abstract}
Let $N_{\mathrm{c}}$ denote the maximum number of electrons that can be bound to an atom of nuclear charge $z$, in the Thomas-Fermi-von Weizsäcker theory. It is proved that $N_{c}$ cannot exceed $z$ by more than one, and thus this theory is in agreement with experimental facts about real atoms. A similar result is proved for molecules, i.e. $N_{\mathrm{c}}$ cannot exceed the total nuclear charge by more than the number of atoms in the molecule.
\end{abstract}

\section{Introduction}

The Thomas-Fermi-von Weizsäcker (TFW) theory (von Weizsäcker 1935, Benguria et al 1981, Lieb 1981) is defined by the energy functional (see Lieb 1981, § VII) (in units in which $\hbar^{2}(2 m)^{-1}=|e|=1$, where $e$ and $m$ are the electron charge and mass)

$\xi(\rho)=A \int\left(\nabla \rho^{1 / 2}(x)\right)^{2} \mathrm{~d} x+\frac{3}{5} \gamma \int \rho(x)^{5 / 3} \mathrm{~d} x-\int V(x) \rho(x) \mathrm{d} x+D(\rho, \rho)$

where

$$
\begin{aligned}
& D(\rho, \rho)=\frac{1}{2} \int \rho(x)|x-y|^{-1} \rho(y) \mathrm{d} x \mathrm{~d} y \\
& V(x)=\sum_{j=1}^{K} z_{j}\left|x-R_{j}\right|^{-1} .
\end{aligned}
$$

Here $z_{1}, z_{2}, \ldots, z_{K} \geqslant 0$ are the charges of $K$ fixed nuclei located at $R_{1}, \ldots, R_{K}$. The total nuclear charge is denoted by $Z, Z=\sum_{j=1}^{K} z_{j} . K=1$ is the atomic case and here we shall simply write $Z=z_{1}=z$. $\mathrm{d} x$ is always a three-dimensional integral. $\xi(\rho)$ is defined for electronic densities $\rho(x) \geqslant 0$ such that each of the terms of $\xi(\rho)$ in (1) is finite. In the physical situation, $\gamma=\gamma_{\text {phys }}=\left(3 \pi^{2}\right)^{2 / 3}$ but, for generality, we shall allow $\gamma$ to be an arbitrary positive constant in what follows. The TFW energy for $N$ (not necessarily an integer) electrons is defined by

$$
E(N)=\inf \left(\xi(\rho) \mid \int \rho=N\right) \text {. }
$$


1046

\section{$R$ Benguria and E H Lieb}

On energetic grounds, the value of $A$ should be chosen to reproduce the Scott term in the expression of the atomic or molecular energy $E(N)$ as a function of $N$ and the nuclear charges, (see Lieb 1981, §§ V.B, VII.D). Numerically one finds (Lieb and Liberman 1982, Lieb 1982), $A=0.1859$. However, we should retain $A$ as an arbitrary positive constant. In the original TFW model (von Weizsäcker 1935) the numerical value of $A$ is 1 .

It is known (Benguria et al 1981, Lieb 1981) that there exists a critical value of $N$ (depending on $A, \gamma$ and the $z_{j}$ and $R_{j}$ ), which we denote by $N_{\mathrm{c}}$, such that for $N \leqslant N_{\mathrm{c}}$ the minimisation problem (4) has a unique solution, whereas for $N>N_{\mathrm{c}}$ there is no solution. In other words, $N_{\mathrm{c}}$ is the maximum number of 'electrons' that can be bound to the atom or molecule. The aim of this paper is to find an upper bound for $N_{\mathrm{c}}$. The value of $N_{\mathrm{c}}$ is given by $\int \rho$, where $\rho \geqslant 0$ is the unique minimising function of $\xi(\rho)$ without constraints. Let $\psi=\rho^{1 / 2}$. Then $\psi$ is the unique positive solution of the TFW equation (for a saturated system),

$$
-A \Delta \psi(x)+\left(\gamma \psi(x)^{4 / 3}-\phi(x)\right) \psi(x)=0
$$

where

$$
\phi(x)=V(x)-|x|^{-1} * \rho \quad \text { with } \rho=\psi^{2} .
$$

Note that (5) is the Euler equation corresponding to the functional $\xi\left(\psi^{2}\right)$. The only previous rigorous results (Lieb 1981, Benguria et al 1981) for $N_{\mathrm{c}}$ were that $Z<N_{\mathrm{c}}<2 Z$.

Our main result is the following.

Theorem 1. For a TFW molecule of $K \geqslant 1$ atoms,

$$
0<N_{\mathrm{c}}-Z \leqslant 270.74(A / \gamma)^{3 / 2} K
$$

for all choices of $z_{1}, \ldots, z_{K}$ and $R_{1}, \ldots, R_{K}$. In particular, for the value of $A$ chosen in Lieb (1981) and Lieb and Liberman (1982) to reproduce the Scott term in the energy (i.e. $A=0.1859)$ and for the physical $\gamma=\left(3 \pi^{2}\right)^{2 / 3}$,

$$
0<N_{\mathrm{c}}-Z<0.7335 \mathrm{~K} \text {. }
$$

In the TFW model the number of electrons is not generally an integer, but in a real atom $N$ and $z$ are required to be integral. How can theorem 1 be interpreted in the light of this additional requirement? One way is the energetic point of view: since $E(N)$ is strictly decreasing for $N<N_{\mathrm{c}}$ and constant for $N \geqslant N_{\mathrm{c}}$ (Lieb 1981, § VII.A), theorem 1 implies that $E(z)>E(z+1)=E(z+2)$. Thus, the $(z+1)$ th electron has a positive binding energy, while the $(z+2)$ th does not, and we can say that a singly ionised atom (but not a doubly ionised atom) is stable. This interpretation, however, suffers from the drawback that there is no solution to (5) when $N=z+1$. A second interpretation that leads to the same conclusion about atomic ionisation, but eliminates the problem that (5) has no solution for $N=z+1$, was kindly provided by John Morgan: introduce the Fermi-Amaldi correction (i.e. replace $D(\rho, \rho)$ in (1) by $(1-$ $1 / N) D(\rho, \rho)$ ). This has the effect of replacing $z$ by $z^{\prime}(N)=N z /(N-1)$. (It also effectively changes $A$ and $\gamma$, but not $A / \gamma$.) Theorem 1 now states that a solution to (5) always exists if $N \leqslant z^{\prime}(N)$ while it never exists if $N-0.74 \geqslant z^{\prime}(N)$. This implies that a solution exists (with $N$ and $z$ integral) if and only if $N \leqslant z+1$. However it is not clear that $E(z+1)<E(z)$ in this Fermi-Amaldi model.

The best previous upper bound on $N_{\mathrm{c}}$ is, as we said, $N_{\mathrm{c}}<2 Z$ (Lieb 1981, theorem 7.23), a result which is valid for both atoms and molecules. It turns out that such a 
bound also holds for the Hartree (bosonic) atom. More recently one of us (Lieb 1984a, Lieb et al 1984) has proved a similar bound for the real Schrödinger equation namely, $N_{\mathrm{c}}<2 z+1$ for an atom and $N_{\mathrm{c}}<2 Z+K$ for a molecule of $K$ atoms. This result (Lieb $1984 \mathrm{a}$, Lieb et al 1984) is valid regardless of the statistics of the bound particles. However, if the bound particles are fermions, as is the case for real matter, $N_{\mathrm{c}}$ should presumably not exceed $z$ (for an atom) by more than one or two electrons. This is still a conjecture; however, it has been proved that $N_{\mathrm{c}} / z \rightarrow 1$ as $z \rightarrow \infty$ for fermions (Lieb et al 1984). On the other hand, we know that $N_{\mathrm{c}}-z>0.2 z$ for the Schrödinger equation of an atom with bosonic particles and for large $z$ (Benguria and Lieb 1983, Baumgartner 1983, 1984). See (Lieb 1984b) for a review of the recent literature on the subject.

In the Thomas-Fermi theory, defined by the energy functional (1) with $A=0, N_{\mathrm{c}}$ is exactly $Z$ even in the molecular case (Lieb 1981, theorem 3.18, Lieb and Simon 1977). Equation (7) implies that for the TFW atom or molecule $N_{\mathrm{c}} \rightarrow Z$ as $A \rightarrow 0$. However, we do not expect $N_{\mathrm{c}}(A)$ to be analytic around $A=0$ because the von Weizsäcker correction is a singular perturbation to Thomas-Fermi theory. It is an open problem to derive an asymptotic expansion for $N_{\mathrm{c}}(A)$ around $A=0$.

Two other open problems arise from the results of this paper. The first is that while we prove an upper bound for $N_{\mathrm{c}}-Z$, we have no lower bound. We conjecture that $N_{\mathrm{c}}-Z \rightarrow$ constant $>0$ as $Z \rightarrow \infty$. The second problem is related to the first: it is highly plausible that $N_{\mathrm{c}}-Z$ is a monotonically increasing function of all the $z_{j}$ (for fixed $\left.R_{1}, \ldots, R_{K}\right)$. Is this true?

This article is organised as follows: in $\S 2$ we give the proof of theorem 1 ; in $\S 3$ we determine the behaviour of $N_{\mathrm{c}}$ as $Z$ goes to zero. Finally in $\S 4$ we give a bound for the chemical potential of a neutral molecule. Such a bound is independent of the charge of the nuclei.

We should like to emphasise that many of the results herein can be extended in two ways: (i) to spherically symmetric 'smeared out' nuclei; (ii) to the TFW theory in which the exponent $\frac{5}{3}$ in (1) is replaced by some $p \neq \frac{5}{3}$ (cf Lieb 1981). For simplicity and clarity we confine ourselves here to point nuclei and $p=\frac{5}{3}$.

\section{Proof of theorem 1}

The proof of theorem 1 will be divided into three steps. First, we estimate the excess charge $Q \equiv N_{\mathrm{c}}-Z$ in terms of the electronic density $\rho$ and the TFw potential $\phi$ evaluated at an arbitrary, but fixed, distance $r$ from all the nuclei. Then we find a local bound for $\rho$ in terms of $\phi$. These two estimates do not involve the $z_{j}$ explicitly. Therefore, if we can prove that at some distance of order one, (i.e. independent of the $z_{j}$ ) the potential $\phi$ is bounded by a constant independent of the $z_{j}$, then the two previous results will imply that $Q$ is less than a constant independent of the $z_{j}$, which is basically what theorem 1 says. Proving this last fact about $\phi$ is our third step. We begin with

Lemma 2. Let $\psi \geqslant 0, \phi$ be the unique solution pair for the TFW equation (5), (6) with $V$ being the potential (3) for a molecule. Then, the function

$$
p(x)=\left(4 \pi A \psi(x)^{2}+\phi(x)^{2}\right)^{1 / 2}
$$

is subharmonic away from the nuclei, i.e. on $\mathscr{R}^{3} \backslash \bigcup_{j=1}^{K} R_{j}$. 
1048

\section{$R$ Benguria and E H Lieb}

Proof. By direct computation

$$
\Delta p=p^{-1}(4 \pi A \psi \Delta \psi+\phi \Delta \phi)+h
$$

with

$$
h=4 \pi A p^{-3}|\phi \nabla \psi-\psi \nabla \phi|^{2} \geqslant 0 .
$$

By (5), (6) the sum of the first two terms in (10) is (away from the nuclei so that $\Delta V=0): 4 \pi \gamma \psi^{10 / 3} \geqslant 0$. Thus, $\Delta p \geqslant 0$, so $p$ is subharmonic.

Remark. Let

$$
W(x) \equiv \gamma \psi(x)^{4 / 3}-\phi(x)
$$

be the 'potential' in the TFW equation (5). Proceeding as in the proof of lemma 2, one can show that

$$
\left(4 \pi A \psi(x)^{2}+W(x)^{2}\right)^{1 / 2}
$$

is subharmonic whenever $W(x) \geqslant 0$.

The next lemma gives a local bound for $\psi$ in terms of $\phi$. This bound is independent of the nuclear charges $z_{j}$.

Lemma 3. For all $\lambda \in(0,1)$ and all $x \in \mathscr{R}^{3}$

$$
\gamma \lambda \psi(x)^{4 / 3} \leqslant \phi(x)+c(\lambda) A^{2} \gamma^{-3}
$$

with

$$
c(\lambda) \equiv(9 / 4) \pi^{2} \lambda^{-2}(1-\lambda)^{-1} .
$$

Proof. Define $u(x) \equiv \psi(x)^{4 / 3}$. Then, from the TFW equation (5),

$$
-\Delta u+(4 / 3 A)(\gamma u-\phi) u+|\nabla u|^{2} / 4 u=0
$$

and hence

$$
-\Delta u+(4 / 3 A)(\gamma u-\phi) u \leqslant 0 .
$$

Also, from (6)

$$
-\Delta \phi=-4 \pi \psi^{2}=-4 \pi u^{3 / 2} \quad x \neq R_{j}, \text { all } j .
$$

Let $v(x)=\gamma \lambda u(x)-\phi(x)-d$, with $d$ a positive constant. We shall show that $v(x) \leqslant 0$, all $x$, for appropriate $d$ and $\lambda$. From equations (15) and (16),

$$
-\Delta v \leqslant-(4 \gamma \lambda / 3 A)(\gamma u-\phi) u+4 \pi u^{3 / 2} \text {. }
$$

Let $S=\{x \mid v(x)>0\} . \psi$ is continuous and goes to zero at infinity; $\phi$ is continuous away from the $R_{j}$ and it also goes to zero at infinity (Benguria et al 1981, § III). Therefore $v$ is continuous away from all the $R_{j}$ and goes to $-d$ at infinity. Hence $S$ is open and bounded. Moreover, $R_{j} \notin S$, all $j$ since $\phi=+\infty$ at the $R_{j}$. On $S, \phi<\gamma \lambda u-d$ so

$$
\begin{aligned}
-\Delta v & \leqslant-(4 \gamma \lambda / 3 A)(\gamma u+d-\gamma \lambda u) u+4 \pi u^{3 / 2} \\
& \leqslant u\left[4 \pi u^{1 / 2}-(4 / 3 A) \gamma^{2} \lambda(1-\lambda) u-(4 / 3 A) \gamma \lambda d\right] \\
& \leqslant 0
\end{aligned}
$$


provided we choose $\lambda \in(0,1)$ and $d=\frac{9}{4}(\pi A / \lambda)^{2} \gamma^{-3}(1-\lambda)^{-1}$ in order that the quantity in brackets [ ] be non-negative for all possible (unknown) values of $u(x)$. With that choice of $\lambda$ and $d, v$ is subharmonic on $S$. On $\partial S v=0$, and therefore $S$ is empty.

Corollary. For all $x \in \mathscr{R}^{3}$

$$
\phi(x) \geqslant-3^{5} 2^{-4} \pi^{2} A^{2} \gamma^{-3} \geqslant-150 A^{2} \gamma^{-3} .
$$

If $x$ is such that $\phi(x) \leqslant 0$, then $p(x)=\left(4 \pi A \psi(x)^{2}+\phi(x)^{2}\right)^{1 / 2}$ satisfies

$$
p(x) \leqslant\left(\frac{4}{3}\right)^{3 / 4} 16 \pi^{2} A^{2} \gamma^{-3} \leqslant 196 A^{2} \gamma^{-3} \text {. }
$$

Proof. By (13) and the fact that $\psi(x)^{4 / 3} \geqslant 0, \phi(x) \geqslant-c(\lambda) A^{2} \gamma^{-3}$ for all $\lambda \in(0,1)$. Minimising $c(\lambda)\left(\right.$ at $\lambda=\frac{2}{3}$ ) gives (17). To prove (18), take $\lambda=\frac{3}{4}$ (which minimises $c(\lambda) / \lambda)$, let $a=-\frac{3}{4} c A^{2} \gamma^{-3}$ and observe from (13) that

$$
p(x)^{2} \leqslant \max _{a \leqslant \phi \leqslant 0}\left[4 \pi A(3 \gamma / 4)^{-3 / 2}(\phi-a)^{3 / 2}+\phi^{2}\right] .
$$

The right-hand side of (19) is convex in $\phi$, so its maximum occurs either at $\phi=0$ or $\phi=a . \phi=0$ prevails and gives (18).

In our next lemma, starting from $\rho$ and $\phi$, we introduce a smeared density $\tilde{\rho}$ and potential $\tilde{\phi}$. We find that $\tilde{\rho}$ and $\tilde{\phi}$ satisfy an inequality resembling the Thomas-Fermi equation for smeared nuclei. Then we use a comparison theorem to get an upper bound for the smeared potential $\tilde{\phi}$ in terms of a universal function (independent of the $z_{j}$ ). Finally, noting that $\phi$ is subharmonic away from the nuclei, we see that essentially the same bound applies to $\phi$. In particular, this lemma says that at distances of order one from all the nuclei, in atomic units, $\phi$ is of order one and, in any case, independent of the $z_{j}$. We note, however, that this bound is not satisfactory both very close and very far from the nuclei. Near the nuclei it diverges too fast. On the other hand, the bound is always positive, whereas $\phi$ is negative at large distances because $Q=N_{\mathrm{c}}-Z$ is strictly positive.

Lemma 4. Let $\psi \geqslant 0, \phi$ be the solution of the TFW equation (5), (6) with $V$ given by (3). Choose any $R>0$ and define

$$
\delta \equiv 25 \pi^{-2} \gamma^{3}=2.53 \gamma^{3}
$$

which is independent of the $z_{j}$. Suppose $x \in \mathscr{R}^{3}$ is such that $\left|x-R_{j}\right|>R$ for all $j=1,2, \ldots, K$. Then

$$
\phi(x) \leqslant A \pi^{2} R^{-2}+\delta \sum_{j=1}^{K}\left(\left|x-R_{j}\right|-R\right)^{-4} .
$$

Proof. Let $W=\gamma \rho^{2 / 3}-\phi, \rho \equiv \psi^{2}$ and consider the Hamiltonian $H=-A \Delta+W . H$ is a non-negative operator, since its ground state, the TFW function $\psi$, has zero energy (chemical potential). Therefore for any function $b \in L^{2}$ with $\nabla b \in L^{2}$ we have

$$
A \int|\nabla b(x)|^{2} \mathrm{~d} x+\int W(x) b(x)^{2} \mathrm{~d} x \geqslant 0 .
$$

We shall choose $b(x)$ to be a translate of the normalised ground state, $e(x)$, of the Laplacian on a ball of radius $R$ with Dirichlet boundary condition. That is, let 
$e(x)=(2 \pi R)^{-1 / 2} \sin \left(\pi|x| R^{-1}\right) /|x|$, for $|x| \leqslant R$ and $e(x)=0$ otherwise. Clearly, $e(x)$ is spherically symmetric, decreasing and it has compact support. Let $b_{x}(y)=e(y-x)$ denote the translate of $e$ and define $g(x)=e(x)^{2}$ and $g_{x}(y)=g(y-x)$. Let $B=$ $A \int\left|\nabla b_{x}(y)\right|^{2} \mathrm{~d} y$. Clearly $B$ does not depend on $x$. With this choice of $b, B=(\pi / R)^{2} A$. From equation (22) we have,

$$
\int W(y) g_{x}(y) \mathrm{d} y \geqslant-B \quad \text { all } x .
$$

Note that $\int W(y) g_{x}(y) \mathrm{d} y=(g * W)(x)$, where an asterisk denotes convolution. Define

$$
\tilde{\phi} \equiv \phi * g-B \text {. }
$$

Since $\phi \in L^{3+\varepsilon}+L^{3-\varepsilon}, \varepsilon>0$, (Benguria et al 1981, proof of lemma 7) and $g \in L^{p}$, all $p \geqslant 1, \tilde{\phi}$ is continuous and goes to $-B$ at infinity (Lieb 1981, lemma 3.1). Using Hölder's inequality, we have for all $x$

$$
\left(g * \rho^{2 / 3}\right)(x) \leqslant[(g * \rho)(x)]^{2 / 3}\left(\int g(y) \mathrm{d} y\right)^{1 / 3}=[(g * \rho)(x)]^{2 / 3}
$$

where we have used $\int g(y) \mathrm{d} y=1$. Let us also define

$$
\tilde{\rho} \equiv g * \rho .
$$

From equations (23)-(26) we obtain for all $x$

$$
B \geqslant(\phi * g)(x)-\gamma\left(\rho^{2 / 3} * g\right)(x) \geqslant \tilde{\phi}(x)+B-\gamma \tilde{\rho}(x)^{2 / 3} .
$$

In other words

$$
\tilde{\phi} \leqslant \gamma \tilde{\rho}^{2 / 3} \text {. }
$$

Notice that $\phi$ is subharmonic away from the nuclei and that $\tilde{\phi}=g * \phi-B$ with $g$ being spherically symmetric, positive, of total mass one and having support in a ball of radius $R$. From this it follows easily that

$$
\phi(x) \leqslant \tilde{\phi}(x)+B
$$

for all $x$ such that $\left|x-R_{j}\right|>R$ (for all $j$ ). Thus, to prove (21) we need a bound on $\tilde{\phi}$.

From equations (6) and (24), using the bound (27) and the fact that the Laplacian commutes with convolution, we compute

$$
-(4 \pi)^{-1} \Delta \tilde{\phi}(x)=\tilde{V}(x)-\tilde{\rho}(x) \leqslant \tilde{V}(x)-\gamma^{-3 / 2}\left[\tilde{\phi}_{+}(x)\right]^{3 / 2}
$$

with

$$
\tilde{V}(x)=\sum_{j=1}^{K} z_{j} g\left(x-R_{j}\right)
$$

and with $\tilde{\phi}_{+}(x)=\max (\tilde{\phi}(x), 0)$.

Note that equation (29) resembles a Thomas-Fermi (TF) equation with smeared nuclei of spherical charge density $z_{j} g\left(x-R_{j}\right)$. Indeed, let $\hat{\phi}$ be the TF potential for this system (i.e. with equality in (29)):

$$
-(4 \pi)^{-1} \Delta \hat{\phi}(x)=\tilde{V}(x)-\gamma^{-3 / 2} \hat{\phi}(x)^{3 / 2} \text {. }
$$

It is known from general TF theory that (31) has a unique solution, $\hat{\phi}$, that goes to zero at infinity. 
It is easy to see that

$$
\tilde{\phi}(x) \leqslant \hat{\phi}(x) \quad \text { for all } x
$$

by observing that if the set $M=\{x \mid \hat{\phi}(x)-\tilde{\phi}(x)<0\}$, then $\hat{\phi}-\tilde{\phi}$ is superharmonic on $M$ and zero on the boundary of $M$ and infinity, so $M$ is empty.

The next step is to bound $\hat{\phi}$. First consider an atom with $V=z / r, r=|x|$, and consider the function $f(r)=\delta(r-R)^{-4}$ which satisfies

$$
(4 \pi)^{-1} \Delta f \leqslant \gamma^{-3 / 2} f^{3 / 2} \quad \text { for } r>R .
$$

Outside the ball of radius $R$ (centred at the origin) $\hat{\phi}$ satisfies

$$
(4 \pi)^{-1} \Delta \hat{\phi}=\gamma^{-3 / 2} \hat{\phi}^{3 / 2} \text {. }
$$

Again, by a comparison argument (and using the fact that $f(r)-\hat{\phi}(r)=\infty$ when $r=R$ )

$$
\hat{\phi}(r) \leqslant f(r) \quad \text { for } r>R .
$$

This, together with (28), proves $(21)$ in the atomic case.

For the molecular case, let $\hat{\phi}_{j}(x)$ be the solution to (31) for an atom of (smeared) nuclear charge $z_{j}$ located at $R_{j}$. By another comparison argument (Lieb and Simon 1977, theorem V.12 or Lieb 1981, corollary 3.6), $\hat{\phi}(x) \leqslant \sum_{j=1}^{K} \hat{\phi}_{j}(x)$. This, together with (35) and (28) proves (21).

We conclude this section with

Proof of Theorem 1: atomic case. Let us start with the atomic case, $V(x)=z /|x|$, in order to expose the ideas most simply. The following facts have been established:

$$
p(x)=\left(4 \pi A \psi(x)^{2}+\phi(x)^{2}\right)^{1 / 2}
$$

is subharmonic for $|x|>0$.

$$
p(x) \leqslant(4 / 3)^{3 / 4} 16 \pi^{2} A^{2} \gamma^{-3}
$$

if $\phi(x) \leqslant 0$.

$$
\phi(x) \leqslant \delta(|x|-R)^{-4}+\pi^{2} A R^{-2}
$$

for all $|x|>R>0$, with $\delta=25 \gamma^{3} \pi^{-2}$ and with arbitrary $R>0$.

$$
\gamma \lambda \psi(x)^{4 / 3} \leqslant \phi(x)+c(\lambda) A^{2} \gamma^{-3}
$$

for all $|x|, 0<\lambda<1$ with $c(\lambda)=9 \pi^{2}\left[4 \lambda^{2}(1-\lambda)\right]^{-1}$.

The functions $p, \phi, \psi$ are functions only of $|x|=r$. As $r \rightarrow \infty, \psi(r) \rightarrow 0$ faster than any power of $r$ (Lieb 1981, theorem 7.24) and $r \phi(r) \rightarrow-Q$. Thus,

$$
r p(r) \rightarrow Q \quad \text { as } \quad r \rightarrow \infty .
$$

The subharmonicity and the fact that $p(r) \rightarrow 0$ as $r \rightarrow \infty$ imply that $r p(r)$ is monotonically decreasing and convex. (This may be seen from the fact that $\Delta p \geqslant 0$ is equivalent, in polar coordinates, to $\mathrm{d}^{2}(r p(r)) / \mathrm{d} r^{2} \geqslant 0$.) Using (40) we conclude that

$$
Q \leqslant r p(r) \quad \text { for any } r>0 .
$$

The same conclusion, (41), can be reached from another viewpoint, which will be important for the molecular case: fix $r>0$ and consider the domain $D_{r}=\{x|| x \mid>r\}$. Let $P$ be any harmonic function on $D_{r}$ with $P(x) \rightarrow 0$ as $|x| \rightarrow \infty$ and $P(x) \geqslant p(x)$ on 
the boundary $|x|=r$. Then $P(x) \geqslant p(x)$ for all $x \in D_{r}$ (Proof: on the set $E=$ $\{x \mid P(x)<p(x)\} \subset D_{r}, p-P$ is positive, subharmonic and $p-P$ vanishes on the boundary of $E$, so $E$ is empty.) Now choose $P(x)=r p(r) /|x|$ for $|x|>r$. Then $r p(r) \geqslant|x| p(x)$, for $|x|>r$. However, $|x| p(x) \rightarrow Q$ as $|x| \rightarrow \infty$, and this establishes (41).

To complete the proof, we make the following specific choices for $r, R, \lambda$ :

$$
r=0.9086\left(\gamma^{3} / A\right)^{1 / 2} \quad R=0.4020\left(\gamma^{3} / A\right)^{1 / 2} \quad \lambda=0.7825 .
$$

If $\phi(r)$ happens to be positive, we use the bound (38), followed by (39) and insert these in (36). (41) then implies that if $\phi(r)>0$ then

$$
Q \leqslant 270.74(A / \gamma)^{3 / 2} \text {. }
$$

(The numbers in (42) were chosen to minimise the coefficient in (43).) On the other hand, if $\phi(r) \leqslant 0$ we can use (37) and (41) to conclude that

$$
Q \leqslant 178.03(A / \gamma)^{3 / 2} \text {. }
$$

Clearly, (43) is the worst case, and this gives theorem 1. Note, however, that if it were to be shown that $\phi(r) \leqslant 0$, then the bound (44) would be valid, and, using the physical values of $A$ and $\gamma$, one would obtain $Q \leqslant 0.49$.

Molecular case. Equation (36) is still valid, except that $p$ is subharmonic only on the set $x \neq R_{j}$ (all $\left.j=1, \ldots, K\right)$. Equations (37) and (39) are also valid. Equation (38) must be replaced by (21) on the set

$$
D_{R}=\left\{x|| x-R_{j} \mid>R \text { for all } j=1, \ldots, K\right\} .
$$

Now choose $r, R$ and $\lambda$ as in (42) and consider the smaller domain

$$
D_{r}=\left\{x|| x-R_{j} \mid>r \text { for all } j=1, \ldots, K\right\} .
$$

Consider the following function which is harmonic on $D_{r}$ :

$$
P(x)=Q_{1} \sum_{j=1}^{K}\left|x-R_{j}\right|^{-1}
$$

where $Q_{1}$ is the right-hand side of (43), namely the value of the upper bound for $r p(r)$ computed in the atomic case under the assumption $\phi(r) \geqslant 0$. As explained above, if we can show that $P(x) \geqslant p(x)$ for all $x$ on the boundary of $D_{r}$, then $P(x) \geqslant p(x)$ for all $x \in D_{r}$. Taking the limit $|x| \rightarrow \infty$ yields

$$
Q=\lim _{|x| \rightarrow \infty}|x| p(x) \leqslant \lim _{|x| \rightarrow \infty}|x| P(x)=K Q_{1}
$$

which is the desired result.

Let $x$ be on the boundary of $D_{r}$, so that $\left|x-R_{j}\right|=r$ for some $j$ (say $j=m$ ). If $\phi(x) \leqslant 0$, the bound (37) is valid and $p(x) \leqslant Q_{2} / r$, where $Q_{2}<Q_{1}$ is the right-hand side of (44). However, $P(x) \geqslant Q_{1}\left|x-R_{m}\right|^{-1}=Q_{1} / r$, so $P(x)>p(x)$. On the other hand, suppose $\phi(x) \geqslant 0$, in which case we can use (21) and (39). Now use proposition 5 below with the choices $t=\frac{3}{2}, s=2$ and

$$
\begin{aligned}
& a_{j}=\delta\left(\left|x-R_{j}\right|-R\right)^{-4} \quad b_{j}=a_{j}(4 \pi A)^{2 / 3} / \gamma \lambda \quad \text { for } j \neq m \\
& a_{m}=\delta\left(\left|x-R_{m}\right|-R\right)^{-4}+A \pi^{2} R^{-2} \\
& b_{m}=\left(a_{m}+c(\lambda) A^{2} \gamma^{-3}\right)(4 \pi A)^{2 / 3} / \gamma \lambda .
\end{aligned}
$$


Recalling that $\left|x-R_{m}\right|=r$ we have

$$
p(x) \leqslant p_{1}(r)+\sum_{j \neq m} \hat{p}\left(x-R_{j}\right)
$$

where $p_{1}(r)$ is precisely the number we calculated before in the atomic case and

$$
\hat{p}\left(x-R_{j}\right)=\left(b_{j}^{3 / 2}+a_{j}^{2}\right)^{1 / 2} .
$$

By construction, $p_{1}(r)=Q_{1} / r$. Thus, $p(x) \leqslant P(x)$ if we can show that $\hat{p}\left(x-R_{j}\right) \leqslant$ $Q_{1} /\left|x-R_{j}\right|$ for $j \neq m$. Let $\left|x-R_{j}\right|=u \geqslant r$. We require that

$$
u^{2}\left[4 \pi A(\gamma \lambda)^{-3 / 2} \delta^{3 / 2}(u-R)^{-6}+\delta^{2}(u-R)^{-8}\right] \leqslant Q_{1}^{2} .
$$

However, the functions $u^{2}(u-R)^{-6}$ and $u^{2}(u-R)^{-8}$ are monotonically decreasing in $u$ for $u>R$. Hence, the left-hand side of (51) is less than its value at $u=r$. But this is obviously less than $r^{2} p_{1}(r)^{2}$ which is $Q_{1}^{2}$.

Proposition 5. Let $0 \leqslant s \leqslant 2,0 \leqslant t \leqslant 2$ and let $a_{1}, \ldots, a_{K}, b_{1}, \ldots, b_{k}$ be $2 K$ non-negative numbers. Then

$$
\left[\left(\sum_{j=1}^{K} a_{j}\right)^{s}+\left(\sum_{j=1}^{K} b_{j}\right)^{t}\right]^{1 / 2} \leqslant \sum_{j=1}^{K}\left(a_{j}^{s}+b_{j}^{t}\right)^{1 / 2} .
$$

Proof. It suffices to prove the proposition for $K=2$ namely, for $a, A, b, B \geqslant 0$,

$$
\left[(a+A)^{s}+(b+B)^{t}\right]^{1 / 2} \leqslant\left(a^{s}+b^{t}\right)^{1 / 2}+\left(A^{s}+B^{t}\right)^{1 / 2} .
$$

If (53) holds then simply take $a=a_{1}, A=\Sigma_{2}^{K} a_{j}$ (and similarly for $b, B$ ) and use induction. Now $(a+A)^{s}=(a+A)^{2} /(a+A)^{2-s} \leqslant\left(a^{2}+2 a A+A^{2}\right) / \max \left(a^{2-s}, A^{2-s}\right) \leqslant$ $a^{s}+2 a^{s / 2} A^{s / 2}+A^{s}$. A similar inequality holds for $(b+B)^{t}$. Squaring (53) and using these inequalities, it suffices to prove that

$$
a^{s / 2} A^{s / 2}+b^{t / 2} B^{t / 2} \leqslant\left(a^{s}+b^{t}\right)^{1 / 2}\left(A^{s}+B^{t}\right)^{1 / 2} .
$$

This, however follows from the Cauchy-Schwarz inequality.

\section{Behaviour of $N_{\mathrm{c}}$ for small $Z$ or small $\gamma$ or large $A$}

Although theorem 1 gives an upper bound for all values of the $z_{j}$, it is primarily useful for the large- $Z$ behaviour of $Q$. In fact, the comparison function $f$ we chose in the proof of lemma 4 , (i.e. $f(x)=\delta(|x|-R)^{-4}$ may be too big when we consider small $z$. Since the atomic $\phi(x)$ is bounded from above by $V(x)=z|x|^{-1}$ and the function $g$ has support on a ball of radius $R$ and total mass $1, \tilde{\phi}(x) \leqslant z|x|^{-1}$ for $|x| \geqslant R$. In particular $\tilde{\phi}(R) \leqslant z|R|^{-1}$, whereas the comparison function $f$ goes to infinity at $|x|=R$. Therefore it is somewhat better to choose $f(x)=\delta(|x|-\alpha R)^{-4}$, where $\alpha=\alpha(z)=1-\left(\delta / z R^{3}\right)^{1 / 4}$ is such that $f(R)=z R^{-1}$. Then, proceeding as in the proof of theorem 1 , one gets a $z_{j}$-dependent bound for $Q$. Although we do not give any details here, we point out that as $z$ goes to zero, for an atom, this upper bound goes to $3.057 A^{3 / 2}$ with $\gamma=\gamma_{\text {phys }}$. We know, however, that as $Z$ goes to zero, $Q$ vanishes because $Q<Z$ (Lieb 1981, theorem 7.23). Thus, the previous bound is not good for small $Z$. Getting the behaviour of $Q$ as a function of $Z$ for small $Z$ is the subject of this section. The main result for $Q$ is contained in equation (63) below. 
We begin with a normalisation convention. Choose $z_{1}^{0}, \ldots, z_{K}^{0}>0$ such that

$$
\sum_{j=1}^{K} z_{j}^{0}=1
$$

and let $R_{1}^{0}, \ldots, R_{K}^{0}$ be fixed, distinct points in $\mathscr{R}^{3}$. Let $Z>0$ be the total nuclear charge in a molecule in which

$$
z_{j}=Z z_{j}^{0} \quad R_{j}=(A / Z) R_{j}^{0} .
$$

In this molecule the length scale is $A / Z$. In TF theory, by comparison, it is $Z^{-1 / 3}$. As $Z \rightarrow 0$ the atoms move apart. One can also treat the case in which the $R_{j}$ remain fixed as $Z \rightarrow 0$; we do not do so explicitly here, but note that in the limit $Z \rightarrow 0$ this is obviously the same as placing all the $R_{j}$ at one common point.

Let us write the solution to (5) as

$$
\psi(x)=Z^{2} A^{-3 / 2} \tilde{\psi}((Z / A) x)
$$

whence

$$
\int \psi(x)^{2} \mathrm{~d} x=Z \int \tilde{\psi}(x)^{2} \mathrm{~d} x .
$$

The TFW equation (5) then reads

$$
\left(-\Delta+\tilde{\gamma} \tilde{\psi}(x)^{4 / 3}-\tilde{\phi}(x)\right) \tilde{\psi}(x)=0
$$

with

$$
\begin{aligned}
& \tilde{\gamma}=\gamma Z^{2 / 3} / A \\
& \tilde{\phi}(x)=\tilde{V}(x)-\left(|x|^{-1} * \tilde{\psi}^{2}\right)(x) \\
& \tilde{V}(x)=\sum_{j=1}^{K} z_{j}^{0}\left|x-R_{j}^{0}\right|^{-1} .
\end{aligned}
$$

With this scaling there is only one non-trivial parameter in the problem, $\tilde{\gamma}$. The potential $\tilde{V}$ is that of a molecule with unit total nuclear charge and $A=1$. Our goal is to elucidate the behaviour of $(58)$ as $\tilde{\gamma} \rightarrow 0$. From now on we shall omit the tilde on the various quantities in (58)-(61).

Formally, at least, as $\gamma \rightarrow 0$ the solution $\psi_{\gamma}$ to (58) approaches the solution $\psi_{\mathrm{H}}$ to the Hartree equation

$$
(-\Delta-\phi(x)) \psi_{\mathrm{H}}=0
$$

with $\phi$ given by (61) and (60) with $\psi_{\mathrm{H}}^{2}$. This equation (which was also used in Benguria and Lieb 1983) has a unique positive solution, $\psi_{\mathrm{H}}$, because the proof in Benguria et al (1981), Lieb (1981), that (1) has a unique minimum and that this minimum is the unique solution to (5) only uses the fact that $\gamma \geqslant 0$. Assuming that $\int \psi^{2} \rightarrow \int \psi_{\mathrm{H}}^{2},(57)$ tells us that

$$
\lim _{Z \rightarrow 0} Q / Z=\int \psi_{\mathrm{H}}^{2}(x) \mathrm{d} x-1 .
$$

Proving (63) is the goal of this section. 
As stated earlier, $1<\int \psi_{\mathrm{H}}^{2}<2$. For the atomic case $K=1, z_{1}^{0}=1$, (62) has been solved numerically (Baumgartner 1983) with the result that

$$
\int \psi_{\mathrm{H}}(x)^{2} \mathrm{~d} x=1.21
$$

Thus, as $Z \rightarrow 0, Q \simeq 0.21 Z$ for an atom. The right-hand side of (63) is not known for a molecule, but we conjecture that

$$
\int \psi_{\mathrm{H}}(x)^{2} \mathrm{~d} x \leqslant 1+0.21 \mathrm{~K} .
$$

The point $\gamma=0$ is not special. We shall prove the following general theorem which says that if $\gamma \rightarrow \Gamma \geqslant 0$ then the solution $\psi_{\gamma} \rightarrow \psi_{\Gamma}$ in a very strong sense. In particular there is strong $L^{2}$ convergence so that (63) is justified.

Theorem 6. Let $\psi_{\gamma}$ (and $\rho_{\gamma}=\psi_{\gamma}^{2}$ ) denote the unique positive solution to the TFW equation (5) for $\gamma \geqslant 0$, with $A$ fixed and with $V$ in equation (3) fixed. (Note: condition (54) is irrelevant here.) Let $\Gamma \geqslant 0$ be fixed. Then, as $\gamma \rightarrow \Gamma, \psi_{\gamma} \rightarrow \psi_{\Gamma}$ in the following senses:

$$
\begin{aligned}
& \nabla \psi_{\gamma} \rightarrow \nabla \psi_{\Gamma} \text { strongly in } L^{2} \text {. } \\
& \psi_{\gamma} \rightarrow \psi_{\Gamma} \text { and } \rho_{\gamma} \rightarrow \rho_{\Gamma} \text { strongly in } L^{p}
\end{aligned}
$$

for all $1 \leqslant p \leqslant \infty$.

$$
|x|^{-1} * \psi_{\gamma}^{2} \rightarrow|x|^{-1} * \psi_{\Gamma}^{2} \text { strongly in } L^{p}
$$

for all $3<p \leqslant \infty$.

$$
D\left(\psi_{\gamma}^{2}, \psi_{\gamma}^{2}\right) \rightarrow D\left(\psi_{\Gamma}^{2}, \psi_{\Gamma}^{2}\right)
$$

$(\mathrm{cf}(2))$.

Proof. Let $\gamma_{n} \geqslant 0$ be any sequence with $\gamma_{n} \rightarrow \Gamma$ and let $\psi_{n} \equiv \psi_{\gamma_{n}}$. Since $\psi_{\Gamma}$ is unique, it suffices to show that some subsequence of $\psi_{n}$ converges to $\psi_{\Gamma}$ in the indicated senses. In the appendix it is proved that $\left\|\psi_{\gamma}\right\|_{\infty}<C_{\infty}=$ constant, independent of $\gamma$. Since $\left\|\psi_{\gamma}\right\|_{2}^{2}<2$, then for all $2 \leqslant p \leqslant \infty$ we have $\left\|\psi_{\gamma}\right\|_{p}<C_{p}=$ constant. With $\xi_{\gamma}$ given by (1) and with $E_{\gamma}$ being the minimum of $\xi_{\gamma}$ we easily find, by considering $\xi_{\gamma}\left(\psi_{\Gamma}^{2}\right)$ and $\xi_{\Gamma}\left(\psi_{\gamma}^{2}\right)$, that

$$
\lim _{\gamma \rightarrow \Gamma} E_{\gamma}=E_{\Gamma}
$$

and also that $\psi_{n}^{2}$ is a minimising sequence for $\xi_{\Gamma}$. By the proof in Lieb (1981) and Benguria et al (1981) of the existence of a minimum for $\xi_{\Gamma}$, and the lower semicontinuity of $\xi_{\Gamma}$, we conclude that there is a subsequence (which we continue to denote by $\psi_{n}$ ) such that

$$
\begin{aligned}
& \nabla \psi_{n} \rightarrow \nabla \psi_{\Gamma} \text { strongly in } L^{2} \\
& D\left(\psi_{n}^{2}, \psi_{n}^{2}\right) \rightarrow D\left(\psi_{\Gamma}^{2}, \psi_{\Gamma}^{2}\right) \\
& \psi_{n} \rightarrow \psi_{\Gamma} \text { almost everywhere }
\end{aligned}
$$

This proves (66) and (69). ((73) follows from the Rellich-Kondrachov theorem.) 
Not only is $\psi_{n}(x) \leqslant C_{\infty}$ for all $x$, but we also have the bound (with some constants $M$ and $\alpha$ )

$$
\psi_{n}(x) \leqslant M \exp \left(-\alpha|x|^{1 / 2}\right)
$$

for $\left|\gamma_{n}-\Gamma\right|$ small enough and for $|x|>R$ for some fixed $R$. To prove (74) we note that for some $R_{1}$ and some $Q>0$

$$
\int_{B} \psi_{\Gamma}(x)^{2} \mathrm{~d} x>Z+Q / 2
$$

with $B=\left\{x|| x \mid<R_{1}\right\}$. Since $H^{1}(B)$ is compactly imbedded in $L^{2}(B)$ (RellichKondrachov), there is a further subsequence such that $\psi_{n} \rightarrow \psi_{\Gamma}$ strongly in $L^{2}(B)$. Hence the entire sequence (see the remark at the beginning of the proof) converges strongly to $\psi_{\Gamma}$ in $L^{2}(B)$. Thus,

$$
\int_{B} \psi_{n}(x)^{2} \mathrm{~d} x>Z+Q / 4
$$

for $n$ large enough. (74) follows by the proof in Benguria et al (1981), lemma 8 or Lieb (1981), lemma 7.24(ii). Consequently, $\psi_{n}(x) \leqslant F(x)$ for all $x$ and $n$ large enough, where $F(x)=C_{\infty}$ for $|x| \leqslant R$ and $F(x)$ is the right-hand side of (74) for $|x|>R$. Since $F \in L^{p}$ for $1 \leqslant p \leqslant \infty$, (67) follows from (73) for $1 \leqslant p<\infty$ by dominated convergence.

The upper bound $F$ and (73) also imply (by dominated convergence) that the convergence in (68) is pointwise almost everywhere and that $g_{\gamma} \equiv|x|^{-1} * \psi_{\gamma}^{2}$ is bounded by a function of the form $G=\min (s, t /|x|)$ for suitable $s$ and $t$. Again, by dominated convergence, we obtain strong convergence in (68) in $L^{p}$ for $3<p<\infty$. The $L^{\infty}$ convergence in (68) follows easily from the $L^{2}$ convergence of $\rho_{\gamma}$ to $\rho_{\Gamma}$ together with the large $|x|$ bound (74).

To prove the $L^{\infty}$ convergence in (67) note that in view of (74) it suffices to prove $L^{\infty}$ convergence on bounded sets. But this follows from the fact (Benguria et al 1981, lemma 7, or Lieb 1981, theorem 7.9) that for any bounded set $S$ and all $x, y \in S$, $\left|\psi_{n}(x)-\psi_{n}(y)\right|<M|x-y|^{1 / 2}$ for some constant $M$ which depends on $S$ but (it is easy to see from the proof) not on $n$.

\section{Lower bound for the chemical potential of a neutral atom or molecule}

Here, we prove a result which is somewhat related to the bound on $N_{\mathrm{c}}$. We shall show that the chemical potential of a neutral system ( $K$ not necessarily one) is bounded from below by a constant independent of the nuclear charge. We conjecture that a similar bound from above should hold.

The chemical potential, $-\mu(N)=\mathrm{d} E / \mathrm{d} N$, as a function of $N$ is nonpositive, continuous and monotonically increasing in $N$ (for fixed nuclear charges) since $E(N)$ is convex in $N$ in TFW theory (Lieb 1981, theorem 7.2 and theorem 7.8(iii)). Therefore the binding energy $\Delta E$ (or affinity) satisfies $|\Delta E|<\mu_{0} Q$, where $Q$ is the added charge ( $Q=1$ for an electron).

The fact that the chemical potential, $-\mu_{0}=-\mu(Z)$, for a neutral atom or molecule is bounded independent of the nuclear charges agrees with what is believed to be the case for the Schrödinger equation. 
Consider the TFW equation for a neutral system (which is a generalisation of (5)), i.e.

$$
-A \Delta \psi+\left(\gamma \psi^{4 / 3}-\phi\right) \psi=-\mu_{0} \psi
$$

with $\phi$ given by (6), (3), or equivalently,

$$
-\Delta \phi=-4 \pi \psi^{2} \quad x \neq R_{i}, i=1,2, \ldots, K .
$$

Here $\int \psi^{2} \mathrm{~d} x=Z=\sum_{i=1}^{K} z_{i}$. Our bound is the following.

Theorem 7. For a neutral system, i.e. for $\int \psi^{2} \mathrm{~d} x=Z$, the chemical potential is bounded from below by,

$$
-\mu_{0} \geqslant-27 \pi^{2} A^{2} \gamma^{-3} \quad \text { all } Z
$$

in the units chosen in the Introduction. In particular, for the value of $A$ chosen in Lieb (1981) to fit the Scott term in the energy, i.e. $A=0.1859$, and with $\gamma=\gamma_{\text {phys }}$, $\mu_{0} \leqslant 0.0105$.

Remark. Since $N_{c}>Z$ (Lieb 1981, theorem 7.19), $\mu_{0}$ is strictly positive.

Proof. First consider the TFW equation with arbitrary $N=\int \psi^{2} \leqslant N_{\mathrm{c}}$, in which case the right side of (76) is replaced by $-\mu(N) \psi$. We know that $\mu(N)=-\mathrm{d} E(N) / \mathrm{d} N$ and that $\mu(N)$ is continuous and monotonically decreasing (Lieb 1981, theorem 7.8(iii)). Therefore (78) will be proved if we can show that for every $N>Z, \mu(N)<27 \pi^{2} A^{2} \gamma^{-3}$. This, we shall now proceed to do.

For every positive $b$ and for all numbers $\psi \geqslant 0$ we have the algebraic inequality

$$
b \psi^{2} \leqslant \gamma \psi^{7 / 3}+d(b) \psi
$$

with

$$
d(b)=27 b^{4} \gamma^{-3} / 256
$$

The TFW equation (with $\int \psi^{2}=N>Z$ ) implies

$$
-A \Delta \psi+b \psi^{2}-\phi \psi \leqslant(d(b)-\mu(N)) \psi
$$

Therefore, if $\mu(N) \geqslant d(b)$

$$
-A \Delta \psi+b \psi^{2}-\phi \psi \leqslant 0
$$

Now, as long as $b$ is chosen so that $b>4(\pi A)^{1 / 2},(77)$ and (81) imply that $\psi<\beta \phi$, all $x \in \mathscr{R}^{3}$, where $\beta$ is the positive root of $b=\beta^{-1}+4 \pi \beta A$. To prove this, let $S=$ $\{x \mid \psi(x)>\beta \phi(x)\}$. Obviously $R_{i} \notin S$. Since $\psi-\beta \phi$ is continuous in $\mathscr{R}^{3} \backslash\left\{R_{i}\right\}, S$ is open. On $S$,

$$
\begin{aligned}
-A \Delta(\psi-\beta \phi) & \leqslant-b \psi^{2}+\phi \psi+4 \pi A \beta \psi^{2} \\
& =\beta^{-1}[\beta \phi-\beta(b-4 \pi A \beta) \psi] \psi \\
& =\beta^{-1}(\beta \phi-\psi) \psi \leqslant 0,
\end{aligned}
$$

where we have used the fact that $b-4 \pi A \beta=\beta^{-1}$. Hence $\psi-\beta \phi$ is subharmonic on $S$. Moreover $\psi-\beta \phi=0$ on $\partial S \cup\{\infty\}$. Therefore $S$ is empty and $\beta \phi(x) \geqslant \psi(x)$ for all $x \in \mathscr{R}^{3}$. Since $\psi \geqslant 0, \phi$ must be non-ncgative everywhere. On the other hand, $\phi=$ 


\section{$1058 \quad R$ Benguria and E H Lieb}

$V-|x|^{-1} * \psi^{2}$ and $\int \psi^{2}>Z$; consequently, $\phi(x)<0$ for sufficiently large $|x|$. This is a contradiction, and we conclude that $\mu(N)<d(b)$ whenever $b=\beta^{-1}+4 \pi \beta A$ for some $0<\beta<\infty$. Choosing $\beta=(4 \pi A)^{-1 / 2}$ yields the desired result, i.e. $\mu(N)<27 \pi^{2} A^{2} \gamma^{-3}$.

Remark. From the asymptotics of the solution of equation (76) we see that $\mu_{0}^{-1 / 2}$ somehow measures the range of the electronic density. If our conjecture is true, such a range would be independent of $Z$.

\section{Acknowledgments}

One of us (RB) would like to thank the Physics Department of Princeton University for their hospitality during the course of this work.

\section{Appendix}

Here, we give a bound for the $L^{\infty}$ norm of the solution to the TFw equation. Such a bound is independent of $\gamma$, the constant in front of the $\psi^{7 / 3}$ term. This bound is used in $\S 3$.

Lemma A.1. Let $\psi$ be the positive solution to the TFW equation for a molecule with $V(x)$ given by (3). Then for all $\gamma>0$

$$
\|\psi\|_{\infty} \leqslant(27 / 16 \pi)^{1 / 2}(Z / A)^{3 / 2}\|\psi\|_{2}
$$

with $\|\psi\|_{2}<(2 Z)^{1 / 2}$ (Lieb 1981, theorem 7.23).

Proof. Because of lemima 9 in Benguria et al (1981), $\psi \in L^{\infty}$. From equation (5), $-A \Delta \psi(x) \leqslant V(x) \psi(x)$. First, consider a single atom with $V(x)=z|x|^{-1}$. In this case, therefore,

$$
A \psi(x) \leqslant(4 \pi)^{-1} \int z|y|^{-1}|x-y|^{-1} \psi(y) \mathrm{d} y
$$

Hence

$$
\begin{aligned}
8 \pi z^{-1} A \psi(x) & \leqslant \int\left(|y|^{-2}+|x-y|^{-2}\right) \psi(y) \mathrm{d} y \\
& =\int|y|^{-2}(\psi(y)+\psi(x-y)) \mathrm{d} y .
\end{aligned}
$$

We decompose this last integral into two terms. One integral over $\{|y|<r\}$ and the other over $\{|y|>r\}$ for any fixed $r>0$. We have,

$$
\begin{aligned}
& \int_{|y|<r}|y|^{-2}(\psi(y)+\psi(x-y)) \mathrm{d} y \leqslant 8 \pi r\|\psi\|_{\infty} \\
& \int_{|y|>r}|y|^{-2}(\psi(y)+\psi(x-y)) \mathrm{d} y \leqslant 2\|\psi\|_{2}(4 \pi / r)^{1 / 2}
\end{aligned}
$$


by Hölder's inequality. Thus, substituting (A.3) and (A.4) into (A.2), we have

$$
8 \pi z^{-1} A \psi(x) \leqslant 8 \pi r\|\psi\|_{\infty}+2\|\psi\|_{2}(4 \pi / r)^{1 / 2} \quad \text { all } r>0
$$

and minimising the right-hand side with respect to $r$ we get

$$
8 \pi A \psi(x) \leqslant 6 z\|\psi\|_{\infty}^{1 / 3}\|\psi\|_{2}^{2 / 3}(2 \pi)^{2 / 3} \quad \text { all } x .
$$

In the molecular case

$$
A \psi(x) \leqslant(4 \pi)^{-1} \sum_{j=1}^{K} \int z_{j}\left|y-R_{j}\right|^{-1}|x-y|^{-1} \psi(y) \mathrm{d} y .
$$

Using the same analysis (A.2)-(A.6) for each term on the right-hand side of (A.7) (but with $\left\{\left|y-R_{j}\right| \gtrless r_{j}\right\}$ and with $r_{j}$ depending on $j$ ) we have that

$$
8 \pi A \psi(x) \leqslant 6 Z\|\psi\|_{\infty}^{1 / 3}\|\psi\|_{2}^{2 / 3}(2 \pi)^{2 / 3} \text {. }
$$

The lemma is proved by taking the supremum over $x$ on the left-hand side of (A.8).

Remark. By making a similar decomposition, one can show that

$$
\left\|B \psi^{2}\right\|_{\infty} \leqslant 3(\pi / 2)^{1 / 3}\|\psi\|_{2}^{4 / 3}\|\psi\|_{\infty}^{2 / 3} \leqslant\|\psi\|_{2}^{2}(Z / A) \times 9 \times 2^{-5 / 3} .
$$

where

$$
\left(B \psi^{2}\right)(x)=\int|x-y|^{-1} \psi(y)^{2} \mathrm{~d} y
$$

and $\|\psi\|_{2}^{2}<2 Z$ (Lieb 1981, theorem 7.23). The second inequality in (A.9) comes from lemma A.1. Actually, the sharp constant in the middle term of (A.9) is $3(\pi / 6)^{1 / 3}$, not $3(\pi / 2)^{1 / 3}$. One can show that the maximising $\rho$ for $\|B \rho\|_{\infty} /\left(\|\rho\|_{1}^{2 / 3}\|\rho\|_{\infty}^{1 / 3}\right)$ is $\rho=$ characteristic function of a ball.

\section{References}

Baumgartner B 1983 Lett. Math. Phys. 7 439-41

1984 J. Phys. A: Math. Gen. 17 1593-602

Benguria R, Brezis H and Lieb E H 1981 Commun. Math. Phys. 79 167-80

Benguria R and Lieb E H 1983 Phys. Rev. Lett. 50 1771-4

Lieb E H 1981 Rev. Mod. Phys. 53 603-41, Errata 198254311

1982 Commun. Math. Phys. 85 15-25
1984a Phys. Rev. Lett. 52 315-7

- 1984b Phys. Rev. A 29 3018-28

Lieb E H and Liberman D A 1982 Numerical Calculation of the Thomas-Fermi-von Weizsäcker Function for an Infinite Atom without Electron Repulsion Los Alamos National Laboratory Report LA 9186-MS

Lieb E H, Sigal I M, Simon B and Thirring W 1984 to be published

Lieb E H and Simon B 1977 Adv. Math. 23 22-116

von Weizsäcker C F 1935 Z. Phys. 96 431-58 
Part V

Stability of Matter 